\title{
Fixation patterns during recognition of personally familiar and unfamiliar faces
}

\section{Goedele van Belle ${ }^{\dagger}$, Meike Ramon*t, Philippe Lefèvre and Bruno Rossion}

Unité de Neurosciences, Cognitives and Laboratoire de Neurophysiologie, Institut des Neurosciences, Université Catholique de Louvain, Louvain-la-Neuve, Belgium

\section{Edited by:}

Tom Hartley, University of York, UK

Reviewed by:

Guillaume A. Rousselet, University of

Glasgow, UK

Gudrun Schwarzer, University of

Giessen, Germany

*Correspondence:

Meike Ramon, Unite Cognition et

Dévelopment, Université Catholique

de Louvain, 10 Place du Cardinal

Mercier, 1348 Louvain-la-Neuve,

Belgium.

e-mail:meike.ramon@uclouvain.be

${ }^{+}$Goedele van Belle and Meike Ramon

contributed equally to this work.
Previous studies recording eye gaze during face perception have rendered somewhat inconclusive findings with respect to fixation differences between familiar and unfamiliar faces. This can be attributed to a number of factors that differ across studies: the type and extent of familiarity with the faces presented, the definition of areas of interest subject to analyses, as well as a lack of consideration for the time course of scan patterns. Here we sought to address these issues by recording fixations in a recognition task with personally familiar and unfamiliar faces. After a first common fixation on a central superior location of the face in between features, suggesting initial holistic encoding, and a subsequent left eye bias, local features were focused and explored more for familiar than unfamiliar faces. Although the number of fixations did not differ for un-/familiar faces, the locations of fixations began to differ before familiarity decisions were provided. This suggests that in the context of familiarity decisions without time constraints, differences in processing familiar and unfamiliar faces arise relatively early - immediately upon initiation of the first fixation to identity-specific information - and that the local features of familiar faces are processed more than those of unfamiliar faces.

Keywords: face recognition, eye movements, personal familiarity

\section{INTRODUCTION}

Recognizing whether a face belongs to a familiar person is an important skill for social behavior. It is, however, still not well known which information human observers use to decide upon familiarity of a face. A common instrument for investigating which information is used to process a visual stimulus is the registration of eye movements (Just and Carpenter, 1976; Goldberg and Wichansky, 2003). Given the inverse relationship between the size of a visual region and the quality of information provided when moving from foveal to peripheral vision (Larson and Loschky, 2009), observers' eye movements are functional in that they allow detailed representation of critical information (Desimone and Duncan, 1995; Egeth and Yantis, 1997). These eye movements involve an alternation between stable gaze positions or fixations, in which the retinal input is processed and the position of the next fixation is determined, and fast movements of the gaze between two fixation positions (Rayner, 1998). Fixation positions are thought to be determined by both the stimulus characteristics and the top-down task requirements (Dodge, 1903; Westheimer, 1954; Yarbus, 1957; Robinson, 1964; Posner, 1980).

In several studies, eye movement recordings have been used to investigate whether there is a difference between the scan patterns for familiar or unfamiliar faces. These studies have usually rendered contradictory results with respect to the influence of familiarity on fixation patterns, with some reporting comparable fixation patterns on facial features for famous and unfamiliar faces (e.g., Stacey et al., 2005), and others suggesting that prior familiarization renders different patterns of fixations (Althoff and Cohen, 1999; Barton et al., 2006; Heisz and Shore, 2008). For instance, Stacey et al. (2005) concluded that when old-new decisions were required, famous and unfamiliar faces did not differ with respect to the sampling of internal features (proportion of fixations, gaze duration). However, their study involved a very coarse definition of "internal features", which comprised a single region encompassing the eyes, nose and mouth, a factor which may account for the fact that their observations are at odds with the findings reported by Althoff and Cohen (1999). These authors found that, when considering the viewing prior to responses provided in a fame decision task, famous faces were associated with less fixations and less regions sampled overall, as well as less fixations on internal features. The results of Barton et al. (2006) support these early findings, in that famous faces were associated with less fixations and shorter scanning duration than unfamiliar ones (which elicited more fixations on the eyes/nose).

However, in a recent study, Hsiao and Cottrell (2008) demonstrated that face recognition (i.e. deciding whether a face has been previously seen, or is novel) could be achieved within only one or two fixations. In their study, participants were familiarized (5-s exposure per face) with a subset of faces, which were subsequently presented in an old/new paradigm. The amount of fixations was explicitly manipulated by removing the stimulus after a predefined number of fixations. They found that performance did not increase any further if observers were allowed to make more than two fixations. Investigation of the fixation pattern showed that these first two fixations were usually located around the top of the nose (see also Orban de Xivry et al., 2008). This initial central location, in between features, suggests that familiarity decisions are based on an appreciation of the face as a whole, rather than detailed local facial information. Indeed, a brain-damaged prosopagnosic patient who is impaired at holistic processing (Ramon et al., 2010; van Belle et al., 2010) rather focused on each facial feature in an analytical way in order to perform a face identification task (Orban de Xivry 
et al., 2008). Hsiao and Cottrell (2008) did not report any difference between the location of the first fixations for familiar(ized) and unfamiliar faces.

There are several reasons that may explain the rather discrepant observations about the pattern of eye gaze fixations for familiar and unfamiliar faces outlined above.

First, one of the possible reasons why the scan patterns for familiar and unfamiliar faces differ across studies might be due to differences considering the definition of "familiar". In some studies, famous faces are used as familiar faces (e.g. Althoff and Cohen, 1999; Stacey et al., 2005; Barton et al., 2006), while in other studies photographs of unfamiliar face stimuli are familiarized (e.g. Henderson et al., 2005; Heisz and Shore, 2008). When familiarized faces are used, identical images are generally used in learning and recognition stages in these studies (see also e.g. Blais et al., 2008). Hence, eye gaze fixations may be driven by pictorial, rather than face identity cues. The same problem may arise for pictures of famous faces, given that the face photographs of famous faces used are often taken from magazines or from the web and have become typical, "iconic", pictures of famous people, who can be recognized based on the particular pose they have on a given photograph (i.e., the iconic photographs of Marylin Monroe, or Che Guevara). In fact, it has been suggested that recognizing these pictures of famous faces does not call upon normal face recognition processes as compared to the recognition of personally familiar faces (Tong and Nakayama, 1999; Knappmeyer et al., 2003; Carbon, 2008). Also using identical images across face learning sessions, Heisz and Shore (2008) showed that differences in fixation patterns occur only after at least four exposures (on consecutive days) to experimentally familiarized faces. It is unclear whether this observation would still hold when comparing unfamiliar with personally familiar faces, which have been learned extensively in real life situations under various viewing conditions and involving multiple views.

A second reason why the scan patterns for familiar and unfamiliar faces may differ across studies is that of the time course of analysis. While Hsiao and Cottrell (2008) did not report any difference between familiarized and unfamiliar face photographs, they concentrated on the first two fixations, which were largely sufficient for fast old/new decisions. In contrast, other studies reported different patterns of fixations for familiar (famous or familiarized) and unfamiliar faces over several seconds, but without providing details about the time-course of eye movement exploration (Althoff and Cohen, 1999; Barton et al., 2006). The question of whether the early and subsequent fixations on faces may differ depending on familiarity thus remains unclear.

Finally, in most of these studies, fixation locations were analyzed using predefined areas of interest (e. g. Henderson et al., 2005; Barton et al., 2006; Stacey et al., 2005), which were rather large, and corresponded roughly to the regions of major internal facial features such as the eyes, nose and mouth. However, recent studies rather suggest that during face recognition tasks fixations on full frontal faces are also preferentially located between features, for instance in between and below the eyes (Blais et al., 2008; Hsiao and Cottrell, 2008; Orban de Xivry et al., 2008; Bindemann et al., 2009). Predefining areas of interest might therefore mask important information about potential differential scan patterns for familiar and unfamiliar faces by reducing the fixation positions to the facial feature that is closest to the actual fixation location. Subtle differences in defining the borders of the areas of interest might also artificially cause differences between the scan patterns in different studies, sometimes leading to conflicting conclusions.

Given these considerations, the present short study was designed to investigate the influence of familiarity on fixation behavior with (a) personally familiar or unfamiliar faces, while (b) analyzing the time-course of fixation, and (c) analyzing fixation locations with higher precision (i.e. without predefined facial areas of interest) than has been done in most previous studies. We recorded participants' eye movements while they engaged in a face recognition task, which required manual familiar/unfamiliar decisions. We scrutinized potential differences in scan patterns for personally familiar as compared to unfamiliar faces in terms of location and duration for each fixation before participants reached a decision. Previous findings indicate that two fixations are sufficient for old-new decisions (Hsiao and Cottrell, 2008), although familiarity decisions under free viewing conditions have been associated with a far greater number of fixations. For this reason, across the sequence of saccades, we were interested in investigating potential differences in scanning (un) familiar faces, which could occur as early as the second fixation.

\section{MATERIALS AND METHODS PARTICIPANTS}

Eleven students (three males) out of a group of about 30 students of the Université catholique de Louvain agreed to participate in the experiment. The group graduated together in 2009 (Master degree in Psychology) and all had been in the same classroom as a small group for about 2 years at the time of testing. Their age ranged from 22 to 24 years, they were all right handed and had normal, or corrected to normal vision. They were financially compensated for their participation. The experiments were undertaken with the understanding and written consent of each subject; the study conforms to The Code of Ethics of the World Medical Association (Declaration of Helsinki).

\section{STIMULI}

Full-front photographs of 26 students from the participants' class were taken to obtain the set of personally familiar face stimuli. The photographs of five male and one female student were excluded from the final set of familiar faces used, due to the presence of facial hair or make-up at the time the photographs were taken. The remaining 20 face stimuli were cropped of external features and hair using Adobe Photoshop. The use of cropped stimuli was favored over uncropped ones, as the presence of hair would have led greater variability in external information in the average face towards which participants were instructed to saccade (see below). Furthermore, previous investigations using uncropped faces indicate that the majority of fixations are located within the face (Althoff and Cohen, 1999; Blais et al., 2008). Note that the only systematic investigation of the effect of experimental familiarization known to us also used cropped faces (Heisz and Shore, 2008), and that these were even more homogenous than ours, which were cropped based on the individual contours and not for instance by means of a generic oval shape (see Figure 1).

Participants were presented with these 20 familiar and an equal amount of unfamiliar faces (matched for eye color and averageluminosity) chosen from a larger database of faces. Importantly, photographs 


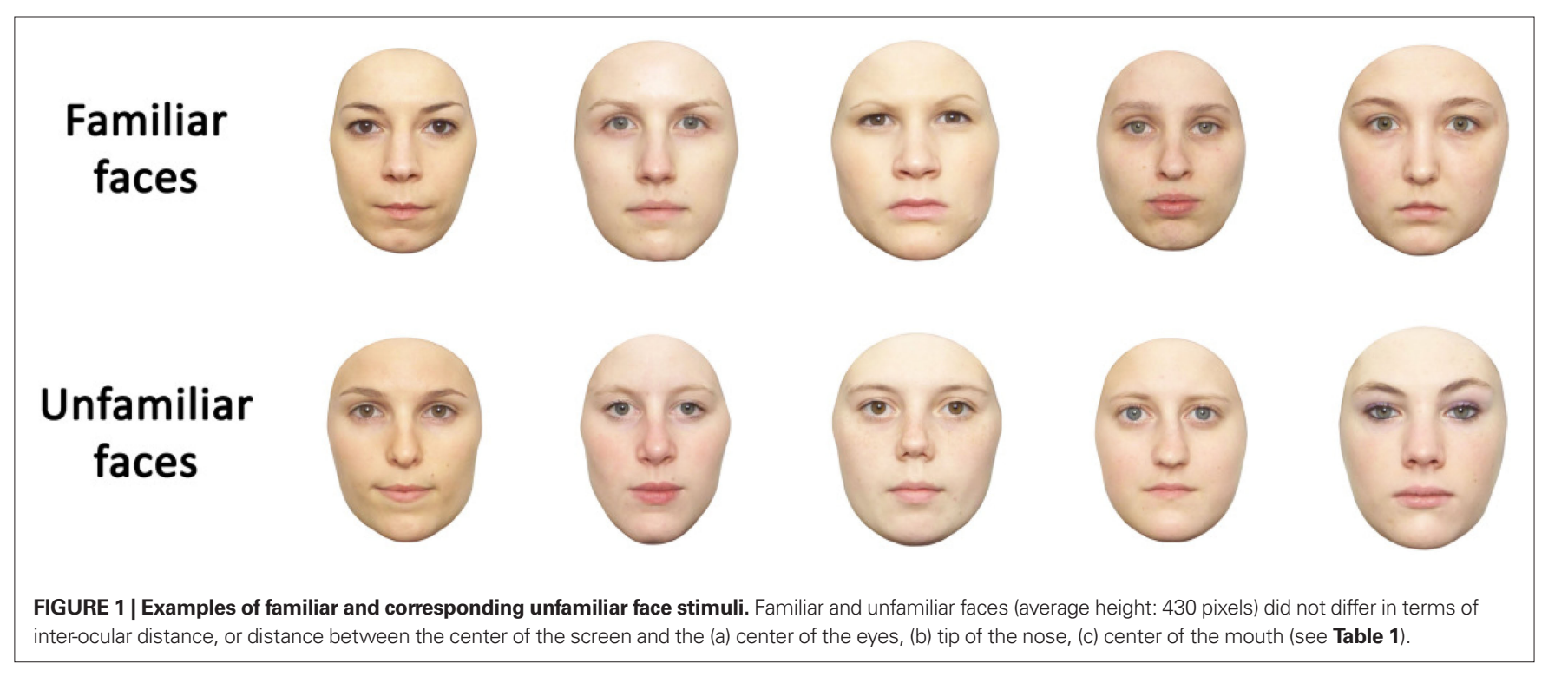

of all face stimuli used were taken under exactly the same conditions (distance, lighting), so that any differences, e.g. in size, reflect natural variations between individuals' faces. Examples of familiar and corresponding unfamiliar faces are provided in Figure 1; permission for publication was obtained from all individuals depicted.

\section{APPARATUS}

The stimuli were displayed using Presentation software, on a 22" Sony Trinitron monitor at a viewing distance of $58 \mathrm{~cm}$ with a spatial resolution of 1400 by 1050 pixels and a refresh rate of $85 \mathrm{~Hz}$. The height of the faces comprised $12^{\circ}$ of visual angle. This roughly corresponds to the size of a real face viewed from a normal conversation distance (Hall, 1966) of $90 \mathrm{~cm}$. Both stimulus display and response registration were handled by an Intel Centrino vPro. Eye movements were registered with an SR Research Eyelink 1000 remote eye tracker at a sampling rate of $250 \mathrm{~Hz}$ and with gaze position error smaller than $0.5^{\circ}$. Head movement was restricted by a chin and head rest.

\section{PROCEDURE}

The basic procedure is illustrated in Figure 2. On each trial, a drift correction with a central fixation cross was followed by a fixation cross on the right of a gray-scale blurred face, consisting of the average of all faces, indicating the position of the stimulus. Participants were instructed to fixate the fixation cross, causing its disappearance, and then to saccade to an average, blurred face. From the moment the participant fixated this blurred average face, it changed into the colored individual face to be recognized. The task was to indicate whether the face was familiar or not, by pressing a right or left key, respectively; half of the trials involved presentation of personally familiar face stimuli. The participant could freely explore the face for a maximum time of $1500 \mathrm{~ms}$. Stimuli disappeared when participants provided manual response, or after the maximum viewing duration.

Note that for a number of reasons in the present investigation the location of the fixation cross was not counterbalanced as was the case in e.g. Blais et al. (2008). On the one hand, although in the literature different methods are used, the locations of first fixations reported are similar (see e.g. Blais et al., 2008; Hsiao and Cottrell, 2008). Randomizing the location of the stimuli or fixation cross would have been preferable had every face been presented four times each (with face or fixation at every possible location). However, we intentionally refrained from presenting the (relatively small sample of) stimuli multiple times, because unfamiliar faces may have become more familiar, reducing differences between familiar and unfamiliar faces. In light of this, varying the location of fixation/faces may have introduced more noise. Importantly, any stereotypical sequences of saccades due to the fixed location would have applied to both groups of faces. As we were particularly interested in differences between these stimulus groups, contrasting conditions would have nullified any such bias, the potential presence of which we therefore do not consider an important issue with respect to our conclusions.

Prior to the actual experiment, participants performed eight practice trials with famous and unknown faces to become acquainted with the procedure. Upon completion of these practice trials (excluded from analysis), participants completed the actual experiment, which involved 80 trials (each of the 20 familiar/unfamiliar faces was presented twice).

\section{ANALYSES}

Participants' accuracy and response times were recorded along with their scan patterns while performing the manual familiarity decision task, and were analyzed to investigate whether performance would differ depending on stimulus type presented ${ }^{1}$.

Heat maps were constructed with the $Z$-values of the relative number of fixations on a given position in the screen for each fixation and condition separately. In order to create these heatmaps, first, a matrix of zeros encompassing the size of the face stimulus was created. For a fixation on a certain pixel, one was added to the value in the matrix on the position of that pixel. Then, to account for the larger region that is processed during fixation of a certain position,

${ }^{1}$ This preference for the left side of the face may be related to the right hemisphere dominance in attentional processes (see e.g. Heilman and Van Den Abell, 1980 Gitelman et al., 1999; Mesulam, 1999; Fink and Heide, 2004), global as compared to local processing (Ellison, et al., 2004), face perception in general (e.g., Hecaen and Angeöergues, 1962; Perrett et al., 1988; Sergent et al., 1992; Zangenehpour and Chaudhuri, 2005), or holistic processing of both unfamiliar and familiar faces (e.g., Parkin and Williamson, 1987; Hillger and Koenig, 1991; Schiltz and Rossion, 2006). 


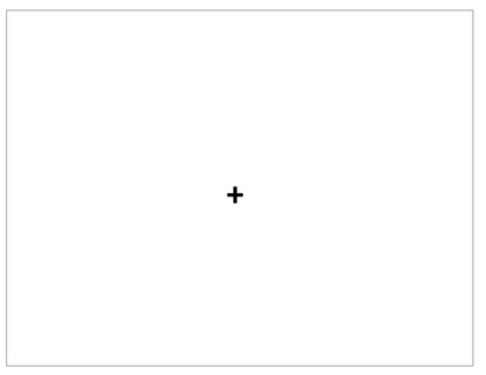

Drift correction

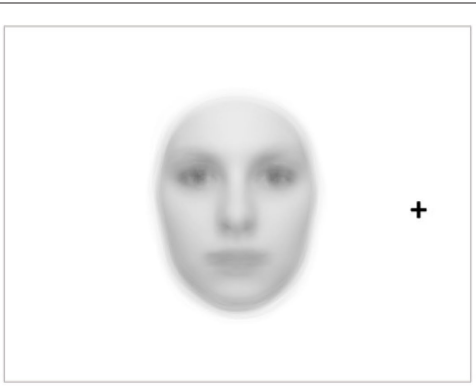

Fixation outside face area

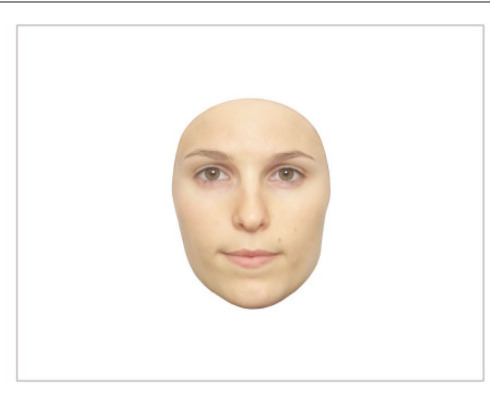

Stimulus (maximum 1500 ms)

FIGURE 2 | Experimental design. After drift correction, participants were presented a fixation cross on the right side of the screen along with an average face, which was replaced by a stimulus upon gaze allocation on the facial area.

fixation positions were smoothed using a Gaussian filter with a sigma of 15 pixels $\left(=0.5^{\circ}\right)$. Only fixations for trials in which participants responded correctly were included. The heat maps were created by standard-normalizing the smoothed values in the matrix using the overall mean and standard deviation of the matrix of pixels. The cluster test proposed by Chauvin et al. (2005) and also used by e.g. Blais et al. (2008) was used for the statistical analysis of the difference between the heat maps of familiar and unfamiliar faces. To this end, pixel-wise $t$-tests were conducted. For each pixel in the matrix, the $t$-value of the difference between the familiar and unfamiliar faces was calculated. Significance of a pixel at the 0.05 level was determined, based on the initial smoothing factor, the size of the cluster to which it belonged, and the $t$-value of the pixel itself.

\section{RESULTS}

As evident from the high accuracy score (average correct responses: $96 \%$ ), participants readily distinguished their classmates' faces from unfamiliar ones despite the absence of extra-facial features. Table 2 summarizes the behavioral findings and the respective statistics. The average response time did not differ significantly across conditions.

The average number of gaze fixations (prior to manual response) made for familiar and unfamiliar face trials did not differ significantly. The distribution of the number of fixations before participants' response is shown in Figure 3. For the large majority (93\%) of the trials with a correct response, 3-6 fixations were sufficient. As illustrated in Figure 4, accuracy was not correlated with the number of fixations on a given trial $(r=0.0058, p=0.29)$.

An ANOVA with "fixation rank" (i.e. their position throughout the sequence of all fixations made on a given trial) and familiarity as the independent variables was conducted in order to investigate their effect on fixation duration. After applying a Bonferroni correction for multiple comparisons in light of the increased prob- ability of Type I error(s) (Familywise Error Rate), no significant main effects were observed (fixation rank: $F(4,40)=2.49, p=0.12$; familiarity: $F(1,10)=0.18, n s)$, and there was no significant interaction between both factors $(F(4,40)=0.99, n s)$.

Regarding the location of fixations, we initially investigated whether the heat maps for unfamiliar/familiar faces including all fixations differed. These heat maps were essentially identical as revealed by no resulting difference when the respective maps were compared.

Figure 5 illustrates the distribution of the gaze position for each individual fixation, for the first five fixations (which constituted $99 \%$ of all fixations), as well as the differences between conditions for each fixation.

Across conditions the first fixation was almost always located within the area in between the eyes, as in previous studies (Hsiao and Cottrell, 2008; Orban de Xivry et al., 2008; Bindemann et al., 2009; see also Cook, 1978; Luria and Strauss, 1978). The second fixation was consistently positioned between the eye located on the left side of the stimulus (Figure 5) and the bridge of the nose. From the third fixation on, the right eye and mouth region were fixated on to a greater extent.

Table 1 | Results of analyses of differences in facial feature positions ( measured in pixels) between familiar and unfamiliar face stimuli ( $\mathrm{df}=38$ ).

\begin{tabular}{lllllll}
\hline & & & & \multicolumn{2}{c}{$\begin{array}{c}\text { Confidence } \\
\text { interval }\end{array}$} \\
\cline { 5 - 6 } & & & & & & \\
\cline { 5 - 6 } & & & & & & \\
\cline { 5 - 6 } & & & & & & \\
Inter-ocular distance & 1.27 & 0.21 & -4.62 & 20.02 & 19 \\
Center of the eyes & 0.08 & 0.93 & -14.60 & 15.85 & 24 \\
Center of the nose & 0.69 & 0.49 & -13.32 & 27.12 & 32 \\
Center of the mouth & 0.70 & 0.49 & -10.65 & 21.85 & 25
\end{tabular}

Table 2 | Mean response time, number of fixations, fixation duration and respective confidence intervals (Cl) for familiar and unfamiliar face stimuli, as well as statistics for comparisons of conditions for each measure.

\begin{tabular}{|c|c|c|c|c|}
\hline & Familiar faces mean [Cl] & Unfamiliar faces mean [Cl] & Difference [Cl] & Statistics \\
\hline Response time & 815 [687, 943] & $930[768,1091]$ & $115[71,162]$ & $t(10)=2.04, p=0.055$ \\
\hline Number of fixations & $4.45[3.77,5.12]$ & $4.47[3.85,5.09]$ & $-0.02[-0.20,0.19]$ & $t(10)=0.11, p=0.69$ \\
\hline Fixation duration & $275[194,357]$ & $278[200,357]$ & $3[-28,34]$ & $F(1,10)=0.18, p=0.68$ \\
\hline
\end{tabular}


Regarding differences in fixation locations between conditions, uncorrected independent comparisons (using the cluster test; see above) yielded no differences at the first fixation. From the second fixation on, with increasing effect size, familiarity-related differences emerged. Note that the lack of difference at the first fixation is an important prerequisite, as the first saccade was always oriented towards an average face derived from all face stimuli, and thus no differences were expected between familiar and unfamiliar face trials. Furthermore, participants were unable to respond before the first fixation.

Complementary analyses of fixation positions weighted by fixation duration were also conducted, as fixation durations are likely to be related to the amount of time used to process information. However, as both analyses rendered highly similar results, we chose to report only the more comprehensible results obtained for fixation locations.

To investigate the effects of stimulus repetition, we conducted further analyses which focused on the effect of presentation (first vs. second) on accuracy scores, RTs, fixation number and fixa-
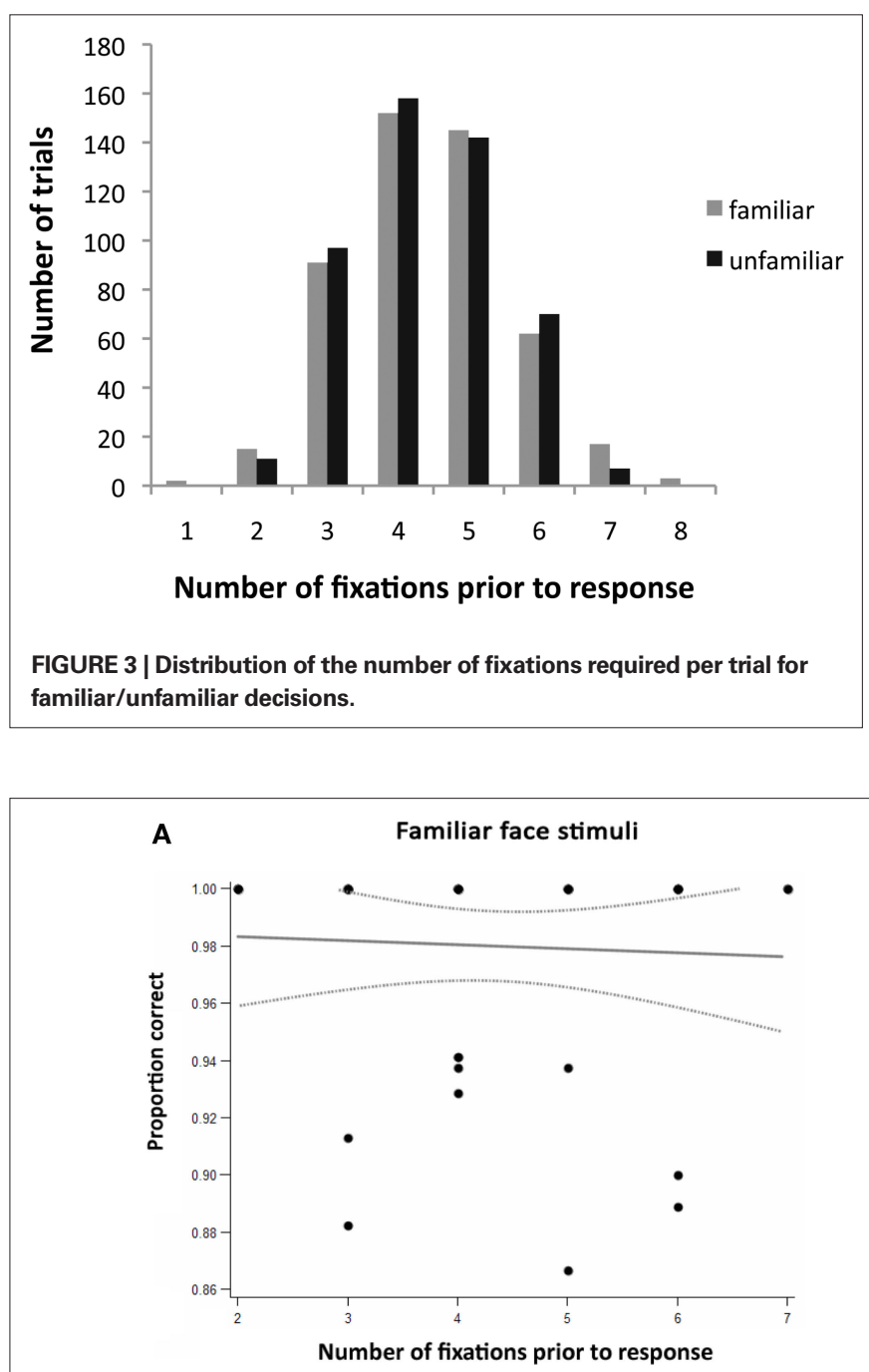

FIGURE 4 | Accuracy as a function of number of fixations prior to recognition, separately for $(A)$ familiar and $(B)$ unfamiliar face stimuli. Dots represent individual participants' average accuracy for a given number of tion duration, respectively. These analyses revealed that neither accuracy $\left(\operatorname{Chi}^{2}(1)=1.34, p=0.25\right)$, nor number of fixations $(t(10)=0.82, p=0.44)$ was influenced by stimulus repetition. Contrariwise, both RTs $(t(10)=9.23, p<0.001)$ and fixation durations $(t(10)=5.37, p<0.001)$ were shorter for the second, as compared to the first stimulus presentation. We further explored this effect in order to determine whether it was driven e.g. by a general learning effect over the course of the experiment, or whether it was explained solely by stimulus repetition. A forward stepwise regression showed a significant effect of trial number $(p<0.001$ for both RT and fixation duration), but no additional effect of presentation (RTs: $p=31$, fixation duration: $p=0.30$ ). Therefore, the effect of stimulus presentation is completely accounted for by a general speeding up over the course of the experiment, most likely to reflect habituation with the task, and not caused by a bias due to stimulus repetition. Presentation-dependent comparisons between fixation locations across conditions were not conducted in light of the small number of participants and sample of stimuli per condition.

\section{DISCUSSION}

The present study analyzed in detail the differences in scan patterns while participants performed familiarity decisions during presentation of unfamiliar and personally familiar faces. Our results confirm recent findings of centrally located initial fixations, positioned just below the eyes, on the top part of the nose (Cook, 1978; Luria and Strauss, 1978; Hsiao and Cottrell, 2008; Orban de Xivry et al., 2008; Bindemann et al., 2009; Sæther et al., 2009), and extend these findings to an old/ new recognition task with personally familiar faces. This position of the first fixation, in between features, may be ideal because it allows normal observers to encode all facial features simultaneously, as a single representation - which is not the case in acquired prosopagnosia (Orban de Xivry et al., 2008; van Belle et al., 2010). Importantly, the initial fixation, directed towards the average face, did not fall on the geometric center of the face stimulus, which would be located below (tip of the nose), but rather on a slightly higher location. This fixation location may correspond to the center of mass (Hsiao and Cottrell,

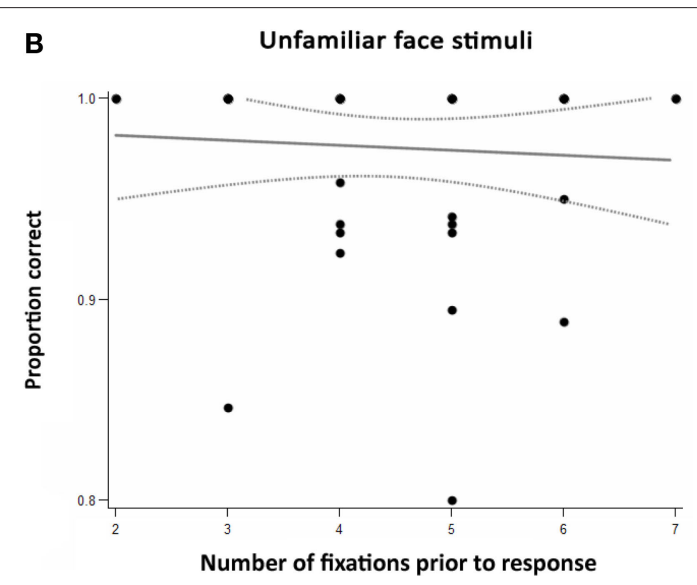

fixations. As indicated by the regression line and confidence intervals, there was no significant correlation between individual accuracy and number of fixations, neither for familiar, nor unfamiliar faces presented. 


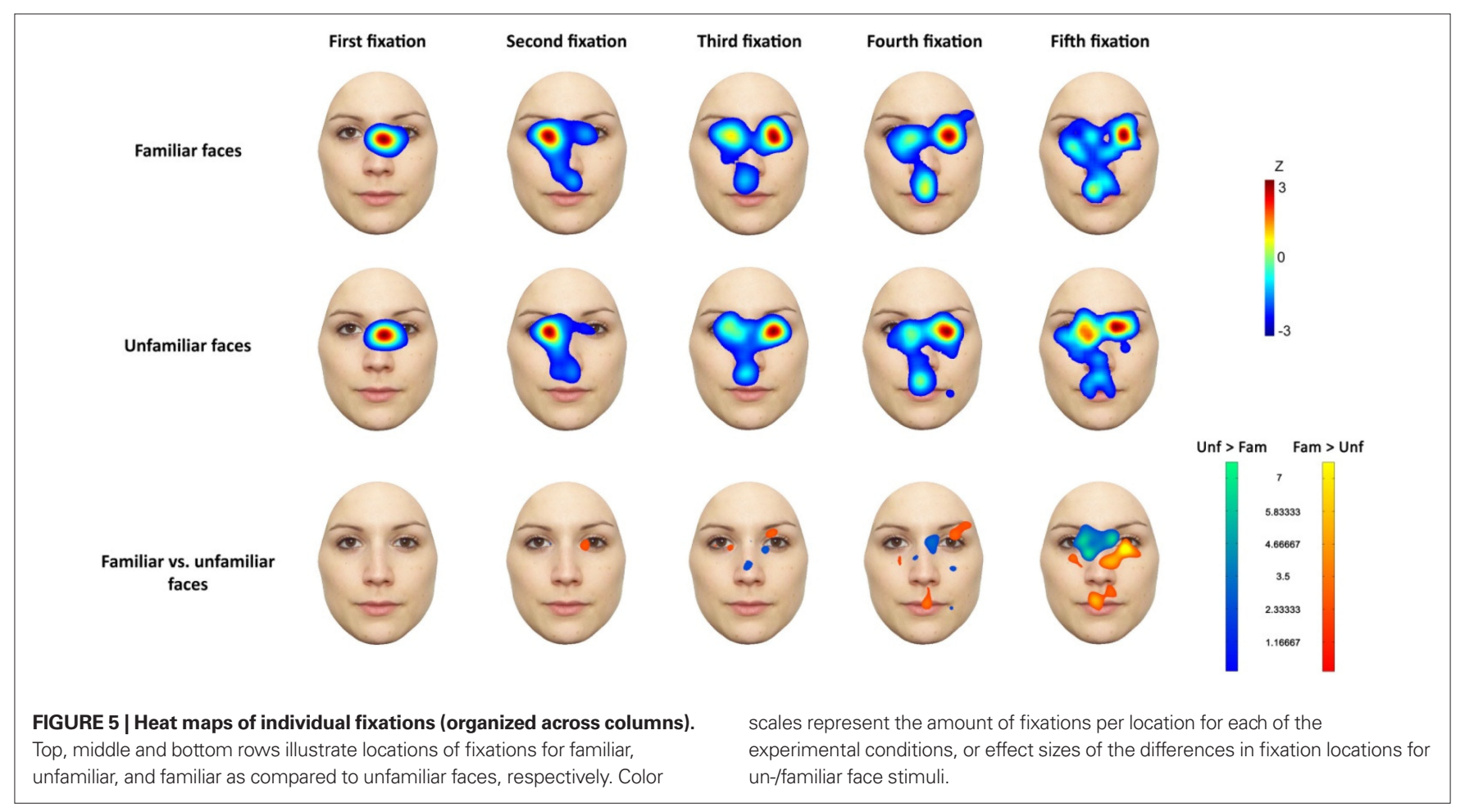

2008; Orban de Xivry et al., 2008) or center of gravity (Bindemann et al., 2009) of the face, i.e. a central position weighted by the amount of diagnostic information of the face. Presumably, this location is fixated because there are higher contrast areas in the top part of the face (eye-eyebrow combination), usually containing more diagnostic information than in the lower part (Davies et al., 1977; Walker-Smith et al., 1977; Shepherd et al., 1981; Haig, 1985; Gosselin and Schyns, 2001; Sekuler et al., 2004).

Independent of familiarity, the second fixation was located on the left side of the face, in between the eye and the nose. This subsequent left eye (of the stimulus) bias (see also e.g. Mertens et al., 1993; Butler et al., 2005; Leonards and Scott-Samuel, 2005; Everdell et al., 2007; Hsiao and Cottrell, 2008) is in agreement with the preference for the left side of the face stimulus (i.e. right side of the face) in face recognition tasks (Gilbert and Bakan, 1973; Gosselin and Schyns, 2001; Brady et al., 2005) $)^{2}$. All subsequent fixations were located predominantly on the eyes and to a lesser extent the space between the nose and the mouth region.

${ }^{2}$ We would like to point out that here - given the nature of the sample tested we were not able to address the question of potential gender differences in scan patterns of personally un-/familiar faces (only 3 males were available for testing). While some authors have reported gender differences in fixations during viewing of static emotional facial expressions (Vassallo et al., 2009), others found sex differences to emerge only when gender was task relevant (Armann and Bülthoff, 2009). In numerous studies gender differences are neglected (and even the sex of participants is not reported, e.g. Williams and Henderson, 2007) despite results indicating behavioral differences in face processing between male and female viewers (e.g. Rehnman and Herlitz, 2006). Further research is thus required to address this issue of gender-dependent familiarity-related processing differences. More generally, because of the original size of the targeted population (classmates), the sample tested was relatively small. Thus, replication of the findings reported here with larger samples in future studies would be desirable.
Long-term familiarity-related processing differences became apparent from the second fixation on, which was the first meaningful one with respect to comparisons across conditions. With number of fixation, the amount of significantly differing clusters increased, as did their respective effect sizes, with most pronounced differences occurring at the latest possible fixation prior to response. Overall, the results suggest that the individual features of familiar faces are processed more (i.e., they receive more fixations overall) than those of unfamiliar ones, and that differences (albeit with smaller effect sizes) can arise relatively early, i.e. from the first meaningful fixation on.

The emergence of differential scan patterns at later stages of the sequence of fixations could be interpreted in the following way. Upon presentation of the average face a saccade is oriented towards the stimulus and its center of mass is fixated, followed by a general left side bias previously demonstrated for face stimuli. Although under normal circumstances familiarity decisions are not yet reached at this point, the visual system may have acquired sufficient information to decide whether a face had been previously seen, or not, if such a decision is required and the visual processing is prematurely terminated (e.g. Hsaio and Cottrell, 2008). This reasoning can account for the emerging differences in fixation locations from this point onwards: all consecutive fixations prior to decision can be considered as reflecting the process of information accumulation, throughout which pre-decisional classification is systematically verified. For unfamiliar faces, fixations are positioned on a location which permits perception of the most salient facial region as a whole, namely between the eyes. For familiar faces, fixations are positioned on the actual diagnostic features (eyes and mouth), with fewer fixations on the center of mass. This pattern of fixation locations could serve to control whether the match between 
the stored representation and the perceived stimulus that activated it is accurate. Presumably, a feature dependent verification process is triggered less for unfamiliar faces, which do not match a representation stored in memory. This interpretation is in line with previous findings of more idiosyncratic and distributed scanning for famous faces (Barton et al., 2006), that have been accounted for in terms of fixations being guided by memory for famous faces, whereas novel face scan patterns reflected more global patterns of information gathering.

Our results are novel in that they demonstrate that - without predefined areas of interest - the sequence of fixations must be taken into account when attempting to identify familiarityrelated processing differences (for a similar approach, albeit with predefined areas of interest see Jack et al., 2009). Comparing the locations of all fixations prior to manual response (taken together) yielded no differences between unfamiliar and personally familiar faces, whereas comparisons at every single fixation did. The present results further demonstrate that processing differences can be observed relatively early - in our case immediately upon availability of identity information. Given that identityspecific information was made available only after (the first) fixation on the blurred average face, the second fixation was thus the first meaningful one. Differences between un-/familiar faces emerged from this point on, albeit more pronouncedly for relatively later fixations. Whether the observed overreliance of processing the individual facial features for familiar face stimuli can be attributed to the more bilateral nature of familiar face representations (as some studies using famous faces suggest, e.g., Kampf et al., 2002; Schweinberger et al., 2003; Baird and Burton, 2008) and thus arises due to a relative lack of left-hemisphere involvement for unfamiliar face stimuli, remains to be determined. The present findings further offer an account that can reconcile the previously reported contradicting findings outlined in the introduction. Given that initial processing stages are characterized by largely overlapping scan patterns, the ability to detect the subsequently emerging familiarity-related processing differences depends largely on the methodological characteristics of a given study. These include the extent to which the representations of the images presented can actually be considered to differ qualitatively, i.e. whether the degree of familiarity

\section{REFERENCES}

Althoff, R. R., and Cohen, N. J. (1999). Eye-movement-based memory effect: a reprocessing effect in face perception. J. Exp. Psychol. Learn Mem. Cogn. 25, 997-1010.

Armann, R., and Bülthoff, I. (2009). Gaze behavior in face comparison: the roles of sex, task, and symmetry. Atten. Percept. Psychophys. 71, 1107-1126.

Baird, L. M., and Burton, A. M. (2008). The bilateral advantage for famous faces: interhemispheric communication or competition? Neuropsychologia 46, 1581-1587.

Barton, J. J., Radcliffe, N., Cherkasova, M. V., Edelman, J., and Intriligator,
J. M. (2006). Information processing during face recognition: the effects of familiarity, inversion, and morphing on scanning fixations. Perception 35, 1089-1105.

Bindemann, M., Scheepers, C., and Burton, A. M. (2009). Viewpoint and center of gravity affect eye movements to human faces. J. Vis. 9, 1-16.

Blais, C., Jack, R. E., Scheepers, C., Fiset, D., and Caldara, R. (2008). Culture shapes how we look at faces. PLoS ONE 3, e3022. doi:10.1371/journal. pone.0003022.

Brady, N., Campbell, M., and Flaherty M. (2005). Perceptual asymmetries are preserved in memory for highly

is sufficient to allow processing differences to be detected. We reason that - by using stimuli derived from faces personally familiar compared to those from faces entirely unfamiliar to the participants - our study circumvented potential limitations regarding this aspect. Furthermore, the spatial resolution inevitably influences the results and thus interpretations offered. For instance, an investigation's sensitivity to detect subtle processing differences will depend largely on whether comparisons are performed between broad, predefined areas-of-interest that vary from one investigation to another, or whether comparisons are performed on the level of pixels comprising an image. Finally, the time period across which eye movements are recorded is likely to determine whether or not familiarity-related processing differences will become apparent. Thus, the duration of (possible) stimulus exploration would influence whether or not they are identified. Furthermore, the fact that past studies have not investigated fixation differences at specific individual time points (in concert with high spatial precision) but rather drew inferences based on data averaged across the entire time course, our study demonstrates that the impact of e.g. stimulus presentation is not to be underestimated.

In conclusion our results demonstrate that prior experience with faces indeed influences fixation patterns in a face recognition task. Furthermore, they emphasize potential disadvantages of predefining regions of interest and the necessity to consider individual fixations rather than averaging across all fixations recorded.

\section{ACKNOWLEDGMENTS}

We thank the subjects for their participation and two reviewers for their insightful comments on an earlier version of the manuscript. Meike Ramon and Bruno Rossion are supported by the Belgian National Fund for Scientific Research (FNRS). This work was supported by grants from the PRODEX program, Fonds National de la Recherche Scientifique, Action de Recherche Concertée (Belgium), and the European Space Agency of the European Union. This paper presents research results of the Belgian Network DYSCO (Dynamical Systems, Control and Optimization), funded by the Interuniversity Attraction Poles Programmes, initiated by the Belgian State, Science Policy Office (Belspo). The scientific responsibility rests with its authors.

familiar faces of self and friend. Brain Cogn. 58, 334-342.

Butler, S., Gilchrist, I. D., Burt, D. M., Perrett, D. I., Jones, E., and Harvey, M. (2005). Are the perceptual biases found in chimeric face processing reflected in eye-movement patterns? Neuropsychologia 43, 52-59.

Carbon, C. C. (2008). Famous faces as icons. The illusion of being an expert in the recognition of famous faces. Perception 37, 801-806.

Chauvin, A., Worsley, K. J., Schyns, P. G., Arguin, M., and Gosselin, F. (2005) Accurate statistical tests for smooth classification images. J. Vis. 5, 659-667.

Cook, M. (1978). "Eye movements during recognition of faces," in Practical
Aspects of Memory, eds M. M. Gruneberg, P. E. Morris, and R. N. Sykes. (New York: Academic Press). 286-292.

Davies, G., Ellis, H., and Shepherd, J. (1977). Cue saliency in faces as assessed by the "Photofit" technique. Perception 6, 263-269.

Desimone, R., and Duncan, J. (1995). Neural mechanisms of selective visual-attention. Annu. Rev. Neurosci. 18, 193-222.

Dodge, R. (1903). Five types of eye movements in the horizontal meridian plane of the field of regard. Am. J. Physiol. 8, 307-329.

Egeth, H. E., and Yantis, S. (1997). Visual attention: control, representation, 
and time course. Annu. Rev. Psychol. 48, 269-297.

Ellison, A., Schindler, I., Pattison, L. L., and Milner, A.D. (2004). An exploration of the role of the superior temporal gyrus in visual search and spatial perception using TMS. Brain 127, 2307-2315.

Everdell, I. T., Marsh, H. O., Yurick, M. D., Munhall, K. G., and Paré, M. (2007). Gaze behaviour in audiovisual speech perception: asymmetrical distribution of face-directed fixations. Perception 36, 1535-1545.

Fink, G. R., and Heide, W. (2004). Spatial neglect. Nervenarzt 75, 389-408.

Gilbert, C., and Bakan, P. (1973). Visual asymmetry in perception of faces. Neuropsychologia 11, 355-362.

Gitelman, D. R., Nobre, A. C., Parrish, T. B., LaBar, K. S., Kim, Y. H., Meyer, J. R., and Mesulam, M. M. (1999). A largescale distributed network for covert spatial attention: Further anatomical delineation based on stringent behavioral and cognitive controls. Brain 122, 1093-1106

Goldberg, J. H., and Wichansky, A.M. (2003). "Eye tracking in usability evaluation: a practitioner's guide," in Mind's Eye: Cognitive and Applied Aspects of Eye Movement Research, eds J. Hyona, R. Radach and H. Deubel. (Amsterdam: Elsevier) 493-516.

Gosselin, F., and Schyns, P. G. (2001). Bubbles: a technique to reveal the use of information in recognition tasks. Vision Res. 41, 2261-2271.

Haig, N. D. (1985). How faces differ--a new comparative technique.Perception 14, 601-615.

Hall, E. T. (1966). The Hidden Dimension. Garden City, NY: Doubleday.

Hecaen, H., and Angeöergues, R. (1962). Agnosia for faces (prosopagnosia). Arch. Neurol. 7, 92-100.

Heilman, K. M., and Van Den Abell, T. (1980). Right hemisphere dominance for attention: the mechanism underlying hemispheric asymmetries of inattention (neglect). Neurology 30, 327-330.

Heisz, J. J., and Shore, D. I. (2008). More efficient scanning for familiar faces. $J$. Vis. 8, 1-10.

Henderson, J. M., Williams, C. C., and Falk, R. J. (2005). Eye movements are functional during face learning. Mem. Cognit. 33, 98-106.

Hillger, L. A., and Koenig, O. (1991). Separable mechanisms in face processing: evidence from hemi- spheric specialization. Special Issue: face perception. J. Cogn. Neurosci. 3 , $42-58$.

Hsiao, J. H., and Cottrell, G. (2008). Two fixations suffice in face recognition. Psychol. Sci. 19, 998-1006.

Jack, R. E., Blais, C., Scheepers, C., Schyns, P. G., and Caldara, R. (2009). Cultural confusions show that facial expressions are not universal. Curr. Biol. 19, 1543-1548.

Just, M. A., and Carpenter, P. A. (1976). Role of eye-fixation research in cognitive psychology. Behav. Res. Methods Instrum. 8, 139-143.

Kampf, M., Nachson, I., and Babkoff, H. (2002). A serial test of the laterality of familiar face recognition. Brain Cogn. 50, 35-50.

Knappmeyer, B., Thornton, I. M., and Bülthoff, H. H. (2003). The use of facial motion and facial form during the processing of identity. Vision Res. 43, 1921-1936.

Larson, A. M., and Loschky, L. C. (2009). The contributions of central versus peripheral vision to scene gist recognition. J. Vis. 9, 1-16.

Leonards, U., and Scott-Samuel, N. E. (2005). Idiosyncratic initiation of saccadic face exploration in humans. Vision Res. 45, 2677-2684.

Luria, S. M., and Strauss, M. S. (1978). Comparison of eye movements over faces in photographic positives and negatives. Perception 7, 349-358.

Mertens, I., Siegmund, H., and Grüsser, O. J. (1993). Gaze motor asymmetries in the perception of faces during a memory task. Neuropsychologia 31, 989-998.

Mesulam, M. M. (1999). Spatial attention and neglect: parietal, frontal and cingulate contributions to the mental representation and attentional targeting of salient extrapersonal events. Philos. Trans. R. Soc. Lond., B, Biol. Sci. 354, 1325-1346.

Orban de Xivry, J. J., Ramon, M., Lefêvre, P., and Rossion, B. (2008). Reduced fixation on the upper area of personally familiar faces following acquired prosopagnosia. J. Neuropsychol. 2(Pt 1), 245-268.

Parkin, A. J., and Williamson, P. (1987). Cerebral lateralisation at different stages of facial processing. Cortex 23, 99-110.

Perrett, D. I., Mistlin, A. J., Chitty, A. J., Smith, P. A., Potter, D. D., Broennimann, R., and Harries, M.
(1988). Specialized face processing and hemispheric asymmetry in man and monkey: evidence from single unit and reaction time studies. Behav. Brain Res. 29, 245-258.

Posner, M. I. (1980). Human visual cognition - Seymour, Phk. Am. J. Psychol. 93, 728-730.

Ramon, M., Busigny, T., and Rossion, B. (2010). Impaired holistic processing of unfamiliar individual faces in acquired prosopagnosia. Neuropsychologia 48, 933-944.

Rayner, K. (1998). Eye movements in reading and information processing: 20 years of research. Psychol. Bull. 124, 372-422.

Rehnman, J., and Herlitz, A. (2007). Women remember more faces than men do. Acta Psychol. (Amst) 124, 344-355.

Robinson, D. A. (1964). Mechanics of human saccadic eye movement. $J$. Physiol. (Lond.) 174, 245-264.

Sæther, L., Belle, W.V., Laeng, B., Brennen, T., and Overvoll, M. (2009). Anchoring gaze when categorizing faces' sex: evidence from eye-tracking data. Vision Res. [Epub ahead of print].

Shepherd, J., Davies, G., and Ellis, H. (1981). "Studies of cue saliency," in Perceiving and Remembering faces, eds G. Davies, H. Ellis and J. Shepherd (London: Academic Press), 105-132.

Schiltz, C., and Rossion, B. (2006). Faces are represented holistically in the human occipito-temporal cortex. Neuroimage 32, 1385-1394.

Schweinberger, S. R., Baird, L. M., Blümler, M., Kaufmann, J. M., and Mohr B. (2003). Interhemispheric cooperation for face recognition but not for affective facial expressions. Neuropsychologia 41, 407-414.

Sekuler, A. B., Gaspar, C. M., Gold, J. M., and Bennett, P. J. (2004). Inversion leads to quantitative, not qualitative, changes in face processing. Curr. Biol. 14, 391-396.

Sergent, J., Ohta, S., and MacDonald, B. (1992). Functional neuroanatomy of face and object processing. A positron emission tomography study. Brain $115,15-36$.

Stacey, P. C., Walker, S., and Underwood, J. D. (2005). Face processing and familiarity: evidence from eye-movement data. Br. J. Psychol. 96, 407-422.

Tong, F., and Nakayama, K. (1999). Robust representations for faces: evidence from visual search. J. Exp.
Psychol. Hum. Percept. Perform. 25, 1016-1035.

Van Belle, G., and de Graef, P., Verfaillie, K., Busigny, T., and Rossion, B. (2010). Whole not hole: Expert face recognition requires holistic perception. Neuropsychologia. doi:10.1016/j. physletb.2003.10.071.

Vassallo, S., Cooper, S. L., and Douglas, J. M. (2009). Visual scanning in the recognition of facial affect: is there an observer sex difference? J. Vis. 9, 1-10.

Walker-Smith, G. J, Gale, A. G., and Findlay, J. M. (1977). Eye movement strategies involved in face perception. Perception 6, 313-326.

Westheimer, G. (1954). Mechanism of Saccadic Eye Movements. Arch. Ophthalmol. 52, 710-724.

Williams, C. C., and Henderson, J. M. (2007). The face inversion effect is not a consequence of aberrant eye movements. Mem. Cognit. 35, 1977-1985.

Yarbus, A. L. (1957). Eye movements during changes in the stationary points of fixation. Biophysics (Oxf.) 2, 679-683.

Zangenehpour, S., and Chaudhuri, A. (2005). Patchy organization and asymmetric distribution of the neural correlates of face processing in monkey inferotemporal cortex. Curr. Biol. 15, 993-1005.

Conflict of Interest Statement: The authors declare that the research was conducted in the absence of any commercial or financial relationships that could be construed as a potential conflict of interest.

Received: 14 January 2010; paper pending published: 18 February 2010; accepted: 25 May 2010; published online: 17 June 2010.

Citation: van Belle G, Ramon M, Leferve P and Rossion B(2010) Fixation patterns during recognition of personally familiar and unfamiliar faces. Front. Psychology 1:20. doi: 10.3389/fpsyg.2010.00020

This article was submitted to Frontiers in Cognitive Science, a specialty of Frontiers in Psychology.

Copyright () 2010 van Belle, Ramon, Lefevre and Rossion. This is an open-access article subject to an exclusive license agreement between the authors and the Frontiers Research Foundation, which permits unrestricted use, distribution, and reproduction in any medium, provided the original authors and source are credited. 